\title{
Alfred Adler aus der Nähe
}

Gabriella Hunziker

\section{«Was ich allerdings glaube, ist, dass wir uns ändern können, wenn wir die Meinung über uns selbst ändern.»}

Korrespondenz:

Dr. med. Gabriella Hunziker Kirchweg 3

CH-9613 Mühlrüti

gabriella.hunziker[at]bluemail.ch
2013 ist erstmals die deutsche Übersetzung von Phyllis Bottomes Adler-Biographie erschienen. Die erste Auflage des Buches der bekannten englischen Schriftstellerin wurde bereits 1939 auf Englisch veröffentlicht. Adler übergab der Autorin, die sein volles Vertrauen besass, sehr persönliches Material in Form von Schriftstücken und mündlichen Berichten. Zwischen Bottome, ihrem Ehemann und Adler entstand ab 1926 eine enge Freundschaft, die bis zu Adlers Tod 1937 bestand. Aus zahlreichen Begegnungen und persönlichen Gesprächen mit Adler und seinen Schülern ist Phyllis Bottomes Adler-Biographie entstanden.

In 32 Kapiteln stellt Bottome Adlers Leben und Lebenswerk dar. Adler betrachtete den Menschen als eine Einheit. Er war davon überzeugt, dass die Persönlichkeit unteilbar ist, weshalb er den Namen Individualpsychologie für seine Wissenschaft wählte. Adler war es wichtig, dass seine Lehre von jedem verstanden wird, weshalb er in allgemein verständlicher Sprache sprach. «Das eigentliche Ziel der Wissenschaft von Seele und Geist des Menschen kann nur sein, dass die menschliche Natur von jedem Menschen verstanden wird. Die Anwendung dieser Wissenschaft soll jeder Menschenseele Frieden bringen.» Adlers bescheidene, uneigennützige und von Mitmenschlichkeit geprägte Persönlichkeit wie auch seine offene und gelassene Einstellung zu Menschen im privaten Bereich und in der Psychotherapie wird von Phyllis Bottome lebendig und treffend beschrie-

ben. Sie belegt dies mit zahlreichen humorvollen und nachdenklich stimmenden Anekdoten Adlers. Humor gehörte zur Psychologie von Alfred Adler. Er empfand Witze und Anekdoten oft hilfreich, um die emotionale Anspannung zu lösen, die manchmal in psychologischen Behandlungen auftrat.

Auch 74 Jahre nach der Erstveröffentlichung hat das Buch nichts an Aktualität verloren. Im Gegenteil, Adlers Denken hat viele moderne Konzepte der Tiefenpsychologie und Psychotherapie beeinflusst und kann noch heute angewendet werden. Eine Erziehung der Kinder im Sinne des Gemeinschaftsgefühls, wie Adler sie fordert, gegründet auf Kooperation statt auf Prestige, kann Nationen und Individuen lehren, sich ohne Aggressionen und Habgier

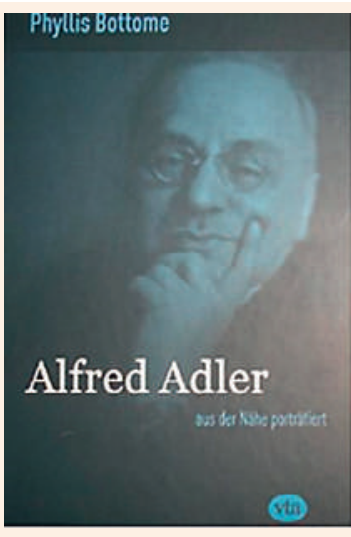

Phyllis Bottome

Alfred Adler aus der Nähe porträtiert

Berlin: VTA-Verlag für Tiefenpsychologie und Anthropologie; 2013.

337 Seiten. $46.90 \mathrm{CHF}$.

ISBN 978-3-00-040056-8

zu begegnen. Adlers Individualpsychologie appelliert an die Menschheit als Ganzes, heute wohl mehr denn je nötig, um den kollektiven Selbstmord zu verhindern.

Die Biographie beginnt mit Adlers Geburt am 7. Februar 1870 in Wien-Penzing, gefolgt von dessen Knaben- und Jugendjahren und ihrem Einfluss auf seine Entwicklung. Adler war sein Leben lang stolz auf seine Jugend, die er unter Strassenbengeln verbrachte. Er lernte dort Achtung vor Mut und Kameradschaft. Der Wunsch, Arzt zu werden, entstand aus dem Erlebnis, als einer seiner Brüder tot neben ihm im Bett lag. In weiteren Kapiteln werden Adlers Beziehung zu den Frauen, sein Berufseinstieg, seine Ansichten zur Religion, das intellektuelle Leben Wiens in den Kaffeehäusern, seine politischen Überzeugungen, der Kreis um Sigmund Freud, die Entwicklung der Individualpsychologie, Adlers Kinder und ihre Erziehung dargestellt, wobei auch Adlers Köchin Sophie zu Wort kommt. Veröffentlicht sind auch Geburtstagsbriefe Adlers an seine älteste Tochter Valentine. Auch ein sehr interessantes Kapitel ist Adlers Umgang mit Kriminellen. Adler hatte einen ehemaligen Sträfling als Gärtner angestellt, aus dem er wieder einen Mitspieler für das soziale Leben gemacht hat. Entscheidend war für ihn die Frage, ob ein Mensch «ein Mitspieler oder ein Spielverderber» war.

Adler stellte nie die Schwierigkeiten seiner Patienten in Abrede. Er zeigte ihnen lediglich - und das in einfühlsamer Weise -, wo sie ihre Schwierigkeiten 
übertrieben und wie mit dem wirklichen Hindernis umzugehen sei. «Sie können Tatsachen nicht ändern», erläuterte er einem Patienten, «aber Sie können den Blick auf sie ändern. Ich habe herausgefunden, dass es immer noch einen weniger schmerzlichen Weg gibt, wenn man scharf genug hinschaut.» Adler war überzeugt, dass der Mensch nicht durch Triebe, Drüsen oder Erbfaktoren gesteuert wird. «Würde ich an eine Besitzpsychologie glauben, gäbe ich die Psychologie ganz auf. Aber ich glaube nicht, dass diese Faktoren bestimmend sind. Was ich allerdings glaube, ist, dass wir uns ändern können, wenn wir die Meinung über uns selbst ändern. Deshalb sehe ich keinen Grund, den Kampf aufzugeben!» Für Adler entsteht der Charakter nicht durch Vererbung, sondern durch die schöpferische Kraft jedes Individuums. «Nicht das ist wichtig, was der Mensch auf die Welt mitbringt, sondern was er daraus macht», so Adler. Im Nachruf von Dr. Lydia Sicher ist Folgendes zu lesen: «Adler lehrte seine Patienten sehen, sich selbst sehen, wie sie waren, aber auch wie sie sein könnten, er lehrte sie den Mut, trotz organischer,
Pädagogik berühmt wurden. Adlers Ansehen wuchs durch diese internationale Anerkennung ausserordentlich. Er wurde in viele Länder eingeladen, um Seminare zum Thema Erziehung zu halten. Auch erhielt Adler eine Stelle als Dozent am Pädagogischen Institut der Stadt Wien. «Lehrer, die einmal nach Adlers Theorien gearbeitet haben, werden nie mehr anders arbeiten können», sagte eine Lehrerin der Autorin. «Sie könnten es nicht, selbst wenn sie es wollten, weil Adler uns lehrte, die Kinder zu verstehen; und kein guter Lehrer könnte diese Haltung seiner selbst und der Kinder wegen aufgeben.» Adler begrüsste jedes Kind warmherzig mit einem Händedruck und behandelte es wie einen Erwachsenen, egal wie alt es war oder wie es sich benahm. In Ferdinand Birnbaums Gedenkrede, gesprochen am 7. Juni 1937, sagte er treffend: «Adler brachte uns bei, mit den $\mathrm{Au}$ gen eines Kindes zu sehen, mit den Ohren eines Kindes zu hören und mit dem Herzen eines Kindes zu fühlen. Wir betrachteten unsere Sorgenkinder nicht länger als unliebsame Störer unserer Lerngemeinschaft ...»

\section{«Mir scheint, was die Welt zurzeit am meisten braucht, ist Gemeinschaftsgefühl.»}

trotz äusserer und innerer Schwierigkeiten ihren Weg zur Realisierung ihres a priori gegebenen Menschenwertes zu gehen, sich einzuordnen und durch die Einordnung ganze Menschen zu werden.» Als Adler 1916 vom Krieg nach Wien zurückkehrte und mit seiner alten Gruppe im Café Central zusammenkam, war es eine bedeutsame Wiederbegegnung, als ein Freund ihn fragte: «Nun, Adler, was gibt's Neues?» Bevor Adler die an ihn gerichtete Frage beantwortete, schaute er mit ernstem Blick von einem zum anderen und sagte dann: «Mir scheint, was die Welt zurzeit am meisten braucht, ist Gemeinschaftsgefühl.» Von diesem Zeitpunkt an war Adler entschlossen, seine Wissenschaft an dieses ethische Ziel zu binden, um die Menschen zu befähigen, mehr Gemeinschaftsgefühl zu entwickeln. Adler hatte beobachtet, dass ein ichbezogener Mensch, der lernt, sich in Richtung des Gemeinschaftsgefühls zu entwickeln, wesentliche Lebensirrtümer korrigieren kann.

Besonders eindrücklich ist das Kapitel über Adlers Erziehungsberatungsstellen, die sich trotz der schrecklichen Armut im Wien der Nachkriegszeit ab 1919 sehr rasch entwickelten und ab 1925 an allen Wiener Hauptschulen existierten, die in der Welt der
Die Adler-Biographie endet mit Adlers Tod im Alter von 67 Jahren in England. Eines der eindrücklichsten Dinge in diesen schweren Stunden, so schreibt die Autorin, war die Natürlichkeit, der Mut und das vernünftige Verhalten der Adler-Familie. Wenige Tage nach Adlers Bestattung trat Adlers zweitälteste Tochter Alexandra Adler an, die Arbeit ihres Vaters fortzusetzen.

Es ist zu wünschen, dass dieses Buch nicht zuletzt auch unter Ärzten weite Verbreitung findet und so die Gedanken der Individualpsychologie für die ärztliche Praxis wieder fruchtbar werden. Eine ganzheitliche, lebensgeschichtliche Betrachtung menschlicher Erkrankungen und Probleme, wie sie uns Adler liefert, würde uns Ärzten zweifellos einen besseren Zugang zu unseren Patienten ermöglichen. Das Buch ist eine hervorragende Einführung in das Lebenswerk Alfred Adlers und ein wertvolles historisches Zeitdokument eines grossen Psychologen, dessen psychologische Erkenntnisse heute noch einen grossen Wert haben. Das Buch trägt dazu bei, Adlers Theorien lebendig zu erhalten und weiterzutragen. Ich kann es nur wärmstens empfehlen. 